PACIFIC JOURNAL OF MATHEMATICS

Vol. 178, No. 2, 1997

\title{
ON CLASSIFICATION OF HEEGAARD SPLITTINGS AND TRIANGULATIONS
}

\author{
DANIEL J. HeATH
}

\begin{abstract}
In this paper we consider Heegaard splittings of 3-manifolds. By using Gabai's concept of thin position on the 1-skeleton of some polyhedral decomposition, together with CassonGordon's concept of strong irreducibility, we prove the Main Theorem (4.0). This theorem will allow us to classify the Heegaard splittings of manifolds whose polyhedral decompositions are particularily nice, which we demonstrate via examples. Specifically, we use it to classify Heegaard splittings of several hyperbolic spaces, including the figure-8 knot complement (Example 6.4) and the genus 2 case of the $5_{2}$-knot complement (Example 6.7).
\end{abstract}

\section{Introduction.}

In this paper, we continue the line of reasoning of [6], resulting in a theorem which we use to classify Heegaard splittings of several hyperbolic spaces. We note that in the spaces mentioned above, the Heegaard splittings coincide with the well-known unknotting tunnels, lending further creedence to the conjecture of $[8]$.

In particular, the Main Theorem essentially says that all Heegaard splittings satisfying a certain technical property for a manifold with a "good" polyhedral decomposition can either be classified or the problem can be simplified in one of two ways. The manifolds arising from the simplification procedure may not be "good," so that a complete classification may not be possible. However, if $M$ is a good manifold with a single boundary component of genus $n$, we can classify its Heegaard splittings of genus $n+1$ using this theorem.

In Section 2 we give the required definitions. We prove some technical lemmas in Section 3, the most important of which (3.4) follows the argument of $([\mathbf{9}, 3.1])$. In Section 4 we prove the Main Theorem. We give an independent result in Section 5; demonstrating that the manifolds having the necessary technical properties form an infinite class.

We give several examples of the use of the Main Theorem in Section 6; we classify Heegaard splittings of several hyperbolic manifolds obtained from 
the cusped census of [11], and discuss the "bad" case. We note that 22 of the manifolds in this census having four or less ideal tetrahedra have easy to find "good" IPD's, and thus that, theoretically, we can apply this theorem to each of them. As the number of exceptional cases is quite large for those with 3 or more edges, we will be content with classifying genus 2 Heegaard splittings in such examples.

This material is based upon work carried out during my tenure as an NSF-nominated Japan Society for the Promotion of Science postdoctoral fellow (NSF grant no. 9400660; JSPS I.D. No. P-94157).

I would like to express my thanks to the department of mathematics at Nara Women's University, where much of this was completed, for its generous hospitality. I would also like to thank Drs. Abigail Thompson and Tsuyoshi Kobayashi for many helpful conversations, and Dr. Jeffrey Weeks for assisting me with the use of SnapPea, his wonderful knot theory computer program.

\section{Definitions.}

Throughout this paper we use the notation $N(*)$ to refer to a regular neighborhood of $*,{ }^{\circ} *$ to refer to the interior of $*$, and the notation $\sharp(*)$ to refer to the number of components of $*$.

For a closed surface $F$, we refer to the number $g$ such that $\chi(F)=2-2 g$ as the genus of $F$. Note that a nonorientable surface may have fractional genus, for example, genus $\left(\mathbf{R} P^{2}\right)=\frac{1}{2}$.

A compression body $H$ is constructed by adding 2-handles to a (closed connected surface) $\times I$ along a collection of disjoint simple closed 2-sided curves on (surface) $\times\{0\}$, and capping off any resulting 2 -sphere boundary components with 3 -balls. The component (surface) $\times\{1\}$ of $\partial H$ is denoted $\partial_{+} H$ and the surface $\partial H \backslash \partial_{+} H$, which may or may not be connected, is denoted $\partial_{-} H$. If $\partial_{-} H=\emptyset$, then $H$ is a handlebody.

For a compact manifold $M$, a 3 -tuple of manifolds $\left(H_{0}, H_{1} ; S\right)$ is called a Heegaard splitting of $M$ if $H_{0}, H_{1}$ is a pair of compression bodies with the property that $M=H_{0} \cup H_{1}$ and $H_{0} \cap H_{1}=\partial_{+} H_{0}=\partial_{+} H_{1}=S$, for some closed connected surface $S$ embedded in $M$. The surface $S$ is called the splitting surface of the Heegaard splitting $\left(H_{0}, H_{1} ; S\right)$, but we shall sometimes refer to either $S$ or $\left(H_{0}, H_{1}\right)$ as the Heegaard splitting. Two Heegaard splittings of $M$ are considered equivalent if their splitting surfaces are isotopic. We note that $\left(H_{0}, H_{1} ; S\right)$ is equivalent to $\left(H_{1}, H_{0} ; S\right)$.

A spine, $X$, of a compression body $H$ is a properly embedded 1-complex such that $H=N\left(\partial_{-} H \cup X\right)$. Let $H$ be a compression body. Once and for all pick a point $p \in{ }^{\circ} H$ and a point $p_{k}$ in each component of $\partial_{-} H$. Let $X$ 
be a spine for $H$ chosen so that:

(0) $X \cap \partial_{-} H=\cup\left\{p_{k}\right\}$,

(1) $X \cap p=\emptyset$ if $H$ is not a handlebody, and

(2) $X \backslash\left(p \cup\left(\cup\left\{p_{k}\right\}\right)\right)$ is a collection of open arcs.

Then we call the number $\sharp(X)$ the complexity of $X$, denoted $c(X)$. It is elementary to check that:

$$
c(X)=\operatorname{genus}\left(\partial_{+} H\right)-\sum \operatorname{genus}\left(\partial_{-} H\right)+\sharp\left(\partial_{-} H\right)-1 .
$$

Let $\left(H_{0}, H_{1}\right)$ be a Heegaard splitting for a manifold $M$. The spinal complexity of $\left(H_{0}, H_{1}\right)$ is the pair $\left\{c\left(X_{0}\right), c\left(X_{1}\right)\right\}$ arranged in lexicographical order. The pair $\{a, b\}$ (with $a \leq b$ ) is said to be less than the pair $\{c, d\}$ (with $c \leq d$ ) if both $a \leq c$ and $b \leq d$, and at least one of the inequalities is strict. A Heegaard splitting $\left(H_{0}, H_{1}\right)$ of $M$ is said to have lower spinal complexity than $\left(\tilde{H}_{0}, \tilde{H}_{1}\right)$ of $\tilde{M}$ if $\left\{c\left(X_{0}\right), c\left(X_{1}\right)\right\}<\left\{c\left(\tilde{X}_{0}\right), c\left(\tilde{X}_{1}\right)\right\}$, see Figure 0 .

An elementary stabilization $E\left(H_{0}, H_{1}\right)$ of $S$ is the splitting surface obtained by taking the connected sum of pairs $(M, S) \sharp\left(S^{3}, T^{2}\right)$, for $T^{2}$ the standard unknotted torus in $S^{3}$. A stabilization of $\left(H_{0}, H_{1}\right)$ is a Heegaard splitting $E^{k}\left(H_{0}, H_{1}\right)$, such that $E^{i}\left(H_{0}, H_{1}\right)$ is an elementary stabilization of $E^{i-1}\left(H_{0}, H_{1}\right)$. A Heegaard splitting is stabilized if it is an elementary stabilization of another splitting. We note that this is equivalent to the existence of proper discs $D_{i} \subset H_{i}$ such that $\partial D_{0} \cap \partial D_{1}=$ \{one point .

Following [9], we will say that a splitting surface is reducible if there exists an essential simple closed (two-sided) curve $c \subset S$ which bounds imbedded discs in both $H_{0}$ and $H_{1}$. A splitting surface is weakly reducible if there exist essential discs $D_{0} \subset H_{0}$ and $D_{1} \subset H_{1}$ with $\partial D_{0} \cap \partial D_{1}=\emptyset$. If $S$ is reducible then it is clearly weakly reducible. If $S$ is not weakly reducible, then $S$ is said to be strongly irreducible. Again, if $S$ is strongly irreducible, $S$ is clearly irreducible.

Remark 2.0. If $S$ is a splitting surface for an irreducible manifold $M$ with genus $(S)>1$, then it is well known that " $S$ is reducible," and " $S$ is stabilized" are equivalent statements. A proof of this can be found in [9].

Since all Heegaard splittings of manifolds of genus $0, \frac{1}{2}$, and 1 are classified by $[\mathbf{1 0}],[5]$, and [1], we shall from this point assume that any manifold in question is of genus at least $\frac{3}{2}$, and thus that "reducible" and "stabilized" are equivalent.

Let $R$ be a closed surface contained in the boundary of a 3-manifold $M$. Let $U_{0}, U_{1}$ be a pair of compression bodies defining a Heegaard splitting of $M$, and assume that $R \subset \partial U_{0}$. Note that there is some $R^{\prime} \subset \partial U_{0}\left(R^{\prime}\right.$ can be empty, or have multiple components) so that $U_{0}=N\left(R \cup R^{\prime}\right) \cup 1$-handles. 
Let $f$ be a homeomorphism $N(R) \rightarrow R \times I$ and $p: R \times I \rightarrow R$ the projection onto the first factor.

Let $M_{1}, M_{2}$ be two manifolds each with non-empty boundary and with Heegaard splittings $\left(U_{0}, U_{1}\right),\left(V_{0}, V_{1}\right)$ respectively. Let $R_{1}, R_{2}$ be two homeomorphic surfaces such that $R_{1} \subset \partial U_{0} \subset \partial M_{1}$ and $R_{2} \subset \partial V_{0} \subset \partial M_{2}$, and let $f_{i}, p_{i}, i=1,2$, be the corresponding functions respectively.

Define an equivalence relation $\sim$ on $M_{1} \cup M_{2}$ as follows:

1) If $x_{i}, y_{i}$ are points such that $x_{i}, y_{i} \in N\left(R_{i}\right)$ and $p_{i} f_{i}\left(x_{i}\right)=p_{i} f_{i}\left(y_{i}\right)$ then $x_{i} \sim y_{i}, i=1,2$.

2) If $x \in R_{1}, y \in R_{2}$ and $g(x)=y$, where $g: R_{1} \rightarrow R_{2}$ is the homeomorphism between the surfaces, then $x \sim y$.

Furthermore we can arrange that the attaching discs on $R_{1} \times I\left(R_{2} \times I\right)$ for the one handles in $U_{0}\left(V_{0}\right)$ respectively, have disjoint images in $R_{1}\left(R_{2}\right)$ and hence do not get identified to each other. Now set:

$$
M=\left(M_{1} \cup M_{2}\right) / \sim, \quad H_{0}=\left(U_{0} \cup V_{1}\right) / \sim, \quad H_{1}=\left(U_{1} \cup V_{0}\right) / \sim .
$$

Note that $H_{0}=V_{1} \cup N\left(R_{1}^{\prime}\right) \cup(1-$ handles $)$ and $H_{1}=U_{1} \cup N\left(R_{2}^{\prime}\right) \cup(1-$ handles) (the 1-handles connect $\partial_{+} V_{1}$ to $\partial N\left(R_{1}^{\prime}\right)\left(\partial_{+} U_{1}\right.$ to $\partial N\left(R_{2}^{\prime}\right)$ respectively)) so that $H_{0}, H_{1}$ are compression bodies defining a Heegaard splitting for M. This Heegaard splitting is called the amalgamation of the Heegaard splittings $\left(U_{0}, U_{1}\right)$ of $M_{1}$ and $\left(V_{0}, V_{1}\right)$ of $M_{2}$ along $R_{1}, R_{2}$. We note that Figure 1 shows the amalgamation process.

Let $M$ be a 3-manifold. Pick a closed subset $\partial_{0} M$ of $\partial M$, and set $\partial_{1} M=$ $\partial M \backslash \partial_{0} M$. Let $\left(H_{0}, H_{1} ; S\right)$ be a genus $g$ Heegaard splitting of $M$ such that $\partial_{-} H_{i}=\partial_{i} M$. Choose a minimal set of defining discs for the compression bodies, so we need at exactly one 3-handle for a handlebody, and none for a compression body which isn't a handlebody.

The handle description defines a Morse function $h: M \rightarrow[0,1]$. The splitting surface will occur as the inverse image of a regular value of $h$. We arrange the singular values of $h$, i.e.

$$
0<a_{1}<\ldots<a_{k}<b_{1}<\ldots<b_{l}<1,
$$

so that passing through a critical point labelled with an $a_{i}$ corresponds to adding a 1 -handle, passing a $b_{i}$ corresponds to adding a 2 -handle, and $h^{-1}(c)$ is isotopic to $S$ for $a_{k}<c<b_{1}$. Thus we have the following:

The leaf of the foliation corresponding to $h^{-1}(0)$ is just $\partial_{-} H_{0}=\partial_{0} M$, if $\partial_{0} M \neq \emptyset$, or the minimal point if $H_{0}$ is a handlebody. All leaves $h^{-1}(r)$ are isotopic for $0<r<a_{1}$, but $h^{-1}\left(a_{1}\right)$ is a singular surface in which two points have been pinched together, forming a 1-handle, see Figure 2. Similarly, each of the $h^{-1}\left(a_{i}\right)$ corresponds to a singular surface in which two points 
on the previous leaves have been pinched together, increasing the genus of the leaves in the foliation by one, or lowering the number of components of $h^{-1}(r)$, so that for $a_{k}<r<b_{1}, h^{-1}(r)$ is a genus $g$ surface. On the other hand, $h^{-1}\left(b_{1}\right)$ is a singular level in which a circle has been pinched into a point, forming a 2-handle, and the picture is the same as in Figure 2, except turned upside down. Then for $b_{1}<r<b_{2}, h^{-1}(r)$ is a genus $g-1$ surface. Note that $h^{-1}(1)$ is the maximal point in $H_{1}$, if $H_{1}$ is a handlebody, or $h^{-1}(1)=\partial_{1} M$ if $\partial_{1} M \neq \emptyset$.

Let $T$ be a polyhedral decomposition for $M$ such that:

(0) $M \backslash(2$-skeleton of $T)$ is a union of balls, $T_{3}^{0}, \ldots T_{3}^{n_{3}}$,

(1) $\partial M$ appears as a union of (punctured) discs in $\partial T_{3}^{k}$,

(2) (1-skeleton of $\mathrm{T}) \backslash \partial M$ is a union of open arcs, $T_{1}^{0}, \ldots T_{1}^{n_{1}}$,

(3) (2-skeleton of $\mathrm{T}) \backslash \partial M$ is a union of discs, $T_{2}^{0}, \ldots T_{2}^{n_{2}}$, such that each $T_{2}^{k}$ is a zerogon (i.e., its boundary lies in $\partial M$ ), a monogon (i.e., its boundary consists of some $T_{1}^{i}$ union a part of $\partial M$ ), a bigon, or a triangle.

Then $T$ is said to be an idealized polyhedral decomposition, or IPD, for $M$.

Denote by $T_{j}=\cup_{k=1}^{n_{j}} T_{j}^{k}$ the $j$-skeleton of $T$, that is, each $T_{3}^{k}$ is a polyhedron, $T_{2}^{k}$ is a zerogon, monogon, bigon, or triangle, and $T_{1}^{k}$ is an arc.

We note that all Heegaard splittings are classified for manifolds $M$ with $T$ having $\sharp\left(T_{1}\right) \leq 1$ (see $[6]$ ), so we assume that $\sharp\left(T_{1}\right)>1$.

Let $I_{1}, \ldots, I_{n}$ be the critical values of $T_{1}$ (with respect to the Morse function $h$ induced by the Heegaard splitting $S$ ), where $0<I_{1}<\ldots<I_{n}<1$. Let $x_{i}$ be regular values of $\left.h\right|_{M},\left.h\right|_{T_{2}}$ such that $0<x_{0}<I_{1}, I_{n}<x_{n}<1$, and $I_{i}<x_{i}<I_{i+1}$ for $0<i<n$. Then each $h^{-1}\left(x_{i}\right)$ is a level surface $S_{i}$. Define the width $w(T)$ of $T$ to be the number of intersections of $\cup S_{i}$ with $T_{1}$, that is, $w(T)=\sum_{i} \sharp\left(S_{i} \cap T_{1}\right)$. If $T$ has been isotoped to have minimal width, we say that $T$ is in thin position. Henceforth we assume that $T$ is in thin position.

If each edge $T_{1}^{k}$ in the 1-skeleton of $T$ has either no critical points, or if all critical points are maxima with respect to the Morse foliation induced by $S$, (or all minima), then $S$ is said to be weakly rigid. If there exists some $i$ so that $T_{1}^{i}$ has more than 1 critical point, and no $T_{1}^{j}$ has exactly one critical point which is a minimum, then $S$ is called a Gabaic Heegaard surface.

Let $M$ be a 3-manifold and $T$ an IDP for $M$. We denote an arc embedded in a face $T_{2}^{k}$ as normal if its endpoints lie on distinct edges of $T_{2}^{k}$. An arc imbedded in a side is abnormal if both its endpoints lie on the same edge. (Arcs with an endpoint on $\partial M$ do not arise in the following arguments.) A curve lying in $T_{2}$ is called simple if it does not intersect $T_{1}$.

Let $r$ be a regular value of $h$ on both $T_{1}$ and $M$. Suppose there is an abnormal arc $\alpha$ of $h^{-1}(r) \cap T_{2}^{k}$. Then $\alpha$ together with a piece $\gamma$ of $T_{1}$ bounds 
a disc $D$ in $T_{2}^{k}$. We say that $D$ is bad if ${ }^{\circ} D \cap h^{-1}(r)$ is empty or consists of simple closed curves. If $D$ is bad, $\gamma$ is above $h^{-1}(r)$ if it lies on the side of $h^{-1}(r)$ containing $h^{-1}(1)$; otherwise it is below. A bad disc lies above or below $h^{-1}(r)$ according to whether $\gamma$ lies above or below, see Figure 3 .

Let $F$ be a closed 2-sided surface embedded in $M$. Assume that $F$ intersects $T_{2}$ in normal arcs and simple curves, and that for at least one $i$, $F \cap T_{1}^{i}>2$. Then $F$ is called a Gabai surface.

If $F$ is a 2-sided surface for which $F \cap T_{2}$ contains only normal arcs and $F \cap T_{3}^{k}$ is a collection of discs for each $k$, then $F$ is called a normalized surface.

We say that a set $S_{1}, \ldots, S_{n}$ of connected, normalized surfaces is a base for $(M, T)$ if any connected normalized surface in $(M, T)$ is represented (up to isotopy preserving number of intersections with each $T_{1}^{i}$ ) by exactly one of the $S_{i}$. If $M$ contains a base with respect to $T$, then $T$ is called a $\operatorname{good}$ IPD, otherwise it is bad. Similarly, a manifold possessing a good IPD is called good, otherwise it is bad.

If $F$ is a 2-sided, closed, incompressible surface in $M$, then $F$ is said to be a Haken surface in $M$. Let $M$ be a manifold, and $F$ a collection of disjoint non-boundary parallel Haken surfaces for $M$. Then the submanifolds $c l(M \backslash N(F))$ are called Haken submanifolds of $M$.

\section{Preliminary Results.}

Theorem 3.0. Let $S$ be a Heegaard splitting surface for the manifold $M$ which is weakly rigid with respect to the idealized polyhedral decomposition $T$. Then $S$ is either a regular neighborhood of a subset of $T_{1}$, together with a neighborhood of some components of $\partial M$, or $S$ is stabilized.

Proof. $[6,2.3]$.

Lemma 3.1 (Thompson). Let $S$ be a Heegaard splitting surface for a 3-manifold $M$. Let $\Delta$ be a collection of compressing discs in $H_{0}$ and set $M^{\prime}=H_{1} \cup N(\Delta)$. If there exists an essential disc $(D, \partial D)$ in $\left(M^{\prime}, \partial M^{\prime} \backslash \partial M\right)$, then $S$ is weakly reducible.

Proof. Note that $M^{\prime}$ has a natural Heegaard splitting along $S$, since to one side we have $H_{1}$, to the other $S \times I$ union some 2-handles. By $[2,1.1]$ we know that if a manifold has a compressible boundary component, then the Heegaard splitting is weakly reducible. Apply this to $\partial M^{\prime}$. Since $\partial M^{\prime}$ is compressible, the Heegaard splitting of $M^{\prime}$ is weakly reducible, and thus $S$ is weakly reducible when considered at the splitting surface for $M$. 
Lemma 3.2 (Scharlemann-Thompson). Suppose that $S$ gives a Heegaard splitting of a 3-manifold $M$ into compression bodies $H_{0}$ and $H_{1}$, and $F \subset S$ is a compact subsurface so that every component of $\partial F$ bounds a disc in $M$ disjoint from ${ }^{\circ} F$. Then either $\partial F$ bounds a collection of discs in a single compression body $H_{i}$, or $S$ is weakly reducible.

Proof. $[9,2.6]$.

Lemma 3.3. Let $F$ be a Haken surface for $M$. Then $F$ can be isotoped so as to be a normalized surface.

Proof. This follows from the argument of $[6,1.1]$.

Lemma 3.4. Let $S$ be a Gabaic Heegaard splitting surface for $M$. Then $S$ is equivalent via isotopy to a Gabai surface.

We will use the following subclaim, which uses the thin position assumption on $T$.

Subclaim 3.5 (Gabai). Let $r$ be a regular value of $h$ on both $T_{1}$ and $M$. Then $h^{-1}(r) \cap T_{2}$ cannot contain abnormal arcs $\alpha_{0}, \alpha_{1}$ cutting off bad discs $D_{0}$ and $D_{1}$ such that $D_{0}$ is above $h^{-1}(r)$ and $D_{1}$ is below.

Proof. $[3, \S 4]$.

Proof of Lemma 3.4. There is at least one minimum on $T_{1}$. Consider the highest such; without loss of generality we may assume that this occurs on $T_{1}^{0}$ at critical level $I_{p}$. $T_{1}^{0}$ has at least one maximum above $I_{p}$. In addition, all arcs of $T_{1}$ above $I_{p}$ contain either a single maximum or no critical points. Note that exchanging the heights of the maxima of two arcs lying above $I_{p}$ does not alter the width of $T_{1}$, see Figure 4 . Thus we may assume that critical level $I_{p+1}$ lying immediately above $I_{p}$ is a maxima for $T_{1}^{0}$.

For every $r$ such that $I_{p}<r<I_{p+1}, T_{1}^{0}$ intersects $h^{-1}(r)$ in at least 3 points. For $r$ very close to $I_{p}, h^{-1}(r) \cap T_{2}$ will contain some bad discs below $h^{-1}(r)$. For $r$ very close to $I_{p+1}, h^{-1}(r) \cap T_{2}$ will contain some bad discs above $h^{-1}(r)$. Again as in [3, $\S 4$, we can conclude that either for some regular value $r$ of $h$ (on $T_{1}, T_{2}$, and $M$ ), $I_{p}<r<I_{p+1}$ there are disjoint bad discs both above and below $h^{-1}(r)$, or that there exists a regular value $r$, $I_{p}<r<I_{p+1}$ such that $h^{-1}(r) \cap T_{2}$ contains no bad discs on either side. By Subclaim 3.5, the first case cannot occur, hence the second case must hold.

Case 1. $a_{k}<r<b_{1}$.

Then $h^{-1}(r)$ is isotopic to $S$, and we have the desired result.

Case 2. $a_{i-1}<r<a_{i}$ for some $i$, or $b_{i}<r<b_{i+1}$. 
The cases are symmetric, so we take $a_{i-1}<r<a_{i}$. Note that $S$ is constructed from $h^{-1}(r)$ by 1-surgery along arcs lying in $h^{-1}((r, 1])$. We can assume that the ends of these arcs are disjoint from $N\left(h^{-1}(r) \cap T_{2}\right)$, so that $h^{-1}(r) \cap T_{2}$ persists into $S$. Also we may assume that these arcs intersect $T_{2}$ transversely, so that no abnormal arcs are added in the process of the 1-surgery. This is the desired result.

\section{Main Result.}

Main Theorem 4.0. Let $T$ be a good IPD for the manifold $M$. Then we can list a finite collection $C$ of (isotopy classes of) surfaces in $M$ together with a finite collection $N$ of 3-submanifolds of $M$ such that if $S$ is a Gabaic Heegaard surface, then one of the following is true:

(a) $S \in C$,

(b) $S$ is induced by a Heegaard splitting $S^{\prime}$ of $M^{\prime} \in N$, and $S^{\prime}$ has lower spinal complexity than $S$,

(c) $S$ is weakly reducible, and thus is obtained from amalgamation of Heegaard splittings of a (some) Haken submanifold(s) of $M$.

Assume that $M$ isn't a compression body, since all Heegaard splittings of compression bodies are classified by [6].

Without loss of generality, we assume that the surface $S$ satisfies the conclusion of Lemma 3.4.

Let $\mu$ be the collection of all normal arcs, and let $U \subset h^{-1}(r)$ be a regular neighborhood of $\mu$ in $h^{-1}(r)$, see Figure 5. Then each component of $\partial U$ is compressible in $M \backslash U$. We call the surface obtained from these compressions $\bar{S}$, and the compressing discs $\Delta$. Note that $\bar{S}$ is a normalized surface, see Figure 6.

By 3.2 we may assume that either all discs in $\Delta$ lie inside of $H_{i}$ for $i=0$ or $i=1$, or that $S$ is weakly reducible.

If $S$ is weakly reducible, then either $S$ is reducible, or by the argument of $[4,1.1], S$ is an amalgamation of Heegaard splittings of Haken submanifolds along a finite collection of Haken surfaces in $M$. We thus assume that $S$ is not weakly reducible.

Let $\bar{H}_{0}, \bar{H}_{1}$ be the manifolds obtained from $H_{0}$ and $H_{1}$ respectively by compression along $\Delta$. Without loss of generality we assume that $\Delta \subset H_{0}$, so that $\bar{H}_{0}=H_{0} \backslash N(\Delta), \bar{H}_{1}=H_{1} \cup N(\Delta)$. Note that $\bar{H}_{0}$ is a union of compression bodies with $\partial_{-} \bar{H}_{0}=\partial_{-} H_{0}$.

We thus consider "all" possible normalized surfaces $\bar{S}$ in $M$ having at least three intersections with some $T_{1}^{i}$. Assume that each such $\bar{S}$ is obtained 
from compression of a Heegaard surface to one side as above. Color the components of $M \backslash \bar{S}$ black and white alternately. Without loss of generality assume that $\bar{H}_{0}$ is black and $\bar{H}_{1}$ is white.

In the following argument, Case $n$ assumes that the hypothesis of Case $i$ is false for $i<n$.

Case $0 . \quad M \backslash \bar{S}$ contains more than one white component.

Then $\bar{H}_{1}$ contains at least two components. But $\bar{H}_{1}=H_{1} \cup N(\Delta)$, implying that $H_{1}$ contained multiple components, a contradiction.

Case 1. $M \backslash \bar{S}$ contains a black component which is not a compression body.

This implies that $\bar{H}_{0}$ is not a union of compression bodies, a contradiction.

Case 2. The white component of $M \backslash \bar{S}$ is not a compression body, and the black component(s) are spineless, i.e., a (perhaps disconnected) surface $\times I$.

Reconstructing $H_{0}$, we must add 1-handles along the cores of the discs $\Delta$. If $\bar{H}_{0}$ contains an $F \times I$ component for which $F$ isn't boundary parallel, then, since $S$ was connected, we can arrange via edge slides (see [4]) that at least one 1-handle runs from $F \times\{0\}$ to $F \times\{1\}$. But $F \times I$ union a 1-handle running between both boundary surfaces isn't a compression body. This in turn implies that $H_{0}$ isn't a compression body, a clear contradiction.

Thus $\bar{S}$ is a collection of boundary parallel surfaces. But $\sharp\left(\bar{S} \cap T_{1}^{i}\right)>2$ for some $i$, so that there are at least two parallel surfaces in $\bar{S}$. Choose one such pair, and call the one closer to the boundary $\bar{S}^{\prime}$. Then to both sides of $\bar{S}^{\prime}$, there is a component of $M \backslash \bar{S}$ homeomorphic to $\bar{S}^{\prime} \times I$. But one of these two must be colored white, contradicting the fact that the white component is not a compression body.

Case 3. The white component of $M \backslash \bar{S}$ is not a compression body, and the black component(s) have non-trivial spine $X_{0}$.

Then we consider the manifold $M^{\prime}=M \backslash N\left(X_{0}\right)$. Note that any Heegaard splitting of $M$ having spine $X_{0}$ induces a Heegaard splitting of $M^{\prime}$, and vice versa. In addition, such an induced Heegaard splitting of $M^{\prime}$ has lower spinal complexity.

Case 4. $M \backslash \bar{S}$ contains more than one black component.

Then $\Delta$ is non-trivial since $H_{0}$ is connected. If $\partial_{+} \bar{H}_{1}$ is compressible, then $S$ was weakly reducible by 3.1 , a contradiction. Thus $\bar{H}_{1}=($ surface $) \times I$, and the original Heegaard splitting can be reconstructed by adding a single 1-handle to $\bar{H}_{0}$ along an arc corresponding to a vertical arc in the I-bundle structure of $\bar{H}_{1}$ by $[4,2.4]$.

Case 5. $\quad \bar{S}$ divides $M$ into two compression bodies. 
If $\bar{S}$ is weakly reducible, let $D_{i} \subset \bar{H}_{i}$ be weakly compressing discs for $\bar{S}$. If $\Delta$ was non-trivial, $D_{1}$ is an essential disc fulfilling the hypothesis of 3.1 , so that $S$ was weakly reducible, a contradiction. Thus we assume that $\Delta=\emptyset$, and thus that $\bar{H}_{i}=H_{i}$. But in this case $\bar{S}=S$, so that $S$ is also weakly reducible, again a contradiction.

Hence $\bar{S}$ isn't weakly reducible. Since $M$ isn't a compression body, there is a non-trivial spine $X_{0}$ for $H_{0}$. As per Case 3, a Heegaard splitting of $M$ having spine $X_{0}$ is induced naturally by a Heegaard splitting of $M^{\prime}=$ $M \backslash N\left(X_{0}\right)$. But $M^{\prime}$ is a compression body, so its Heegaard splittings have already been classified by $[4,2.5]$.

Note that if $\bar{S}$ contains three parallel surfaces, we may apply Case 0 . If there exists a finite base for $(M, T)$, there are only finitely many normalized surfaces not containing a parallel trio. Thus, in applying the above argument, we only need to check finitely many surfaces. For each such surface, there are two possible colorings, and thus finitely many possibile outcomes.

This is the desired result.

\section{Good Idealized Polyhedral Decompositions.}

Let $F$ be a surface intersecting $T_{2}$ in normal arcs and $T_{3}$ in discs. We shall denote $F$ by the $n$-tuple $\left(\sharp\left(F \cap T_{1}^{0}\right), \ldots, \sharp\left(F \cap T_{1}^{n-1}\right)\right)$. Note that any normalized surface has a unique notation (modulo isotopy preserving number of intersections with each $T_{1}^{i}$ ) as such an $n$-tuple. Conversely, any such $n-$ tuple either represents a normalized surface, a 1-sided surface, or else no surface $F$ with the above properties exists, see Figure 7 .

Theorem 5.0. Let $M$ be a 3-manifold, and assume that $M$ has an IPD with only one edge $T_{1}^{0}$. Then $(M, T)$ is good.

Proof. This follows from the argument of $[6,1.1]$.

Theorem 5.1. Let $M$ be a 3-manifold, and assume that $M$ has an IPD having two edges $T_{1}^{0}, T_{1}^{1}$. Then $(M, T)$ is good.

Proof. We denote each surface $F$ intersecting $T_{2}$ in normal $\operatorname{arcs}$ and $T_{3}$ in discs with a pair of non-negative integers $(a, b)$ as above.

Such surfaces $F$ cannot intersect zerogonal faces, so we may assume that no such faces exist.

If a monogonal face exists, say with edge $T_{1}^{1}$, then $\sharp\left(F \cap T_{1}^{1}\right)=0$, so we may for the purposes of the argument remove this edge from the manifold, and consider the manifold $M^{\prime}=M \backslash N\left(T_{1}^{1}\right)$, which has an IPD with only one edge. Then we apply Theorem 5.0 to $\left(M^{\prime}, T^{\prime}\right)$, and pull the argument 
back into $M$ in the natural way. We may thus assume that there are no monogonal faces in $T$.

If $T$ contains a bigonal face with both $T_{1}^{0}$ and $T_{1}^{1}$ edges, then $\sharp\left(F \cap T_{1}^{0}\right)=$ $\sharp\left(F \cap T_{1}^{1}\right)$. We may thus for the purposes of this argument assume that $T$ has only one edge, and apply 5.0. Thus we assume that $T$ contains no bigonal face with different edges.

Any other bigonal faces give no information concerning normalized surfaces, so we may ignore them for the purposes of this argument. We then assume that all faces of $T$ are triangular. A face with $(i)-T_{1}^{0}$ edges and $(j)-T_{1}^{1}$ edges we denote $[\mathrm{i}, \mathrm{j}]$. Note that $j=3-i$, so there are four types of faces: $[0,3],[1,2],[2,1]$, and $[3,0]$, see Figure 8 .

The case when all faces are of type $[0,3]$ or $[3,0]$ is contained in 5.0. We thus assume we have faces of type:

Case 0. [1,2] only.

Then $(0,1)$ and $(2,1)$ are either normalized surfaces or 1-sided. (A simple linear algebra argument shows that such $F$ exist.) Assume the former. Any normalized surface can be denoted $(2 n, m)$, where $m \geq n$, since each face in $T_{2}$ looks like Figure 9. A normalized surface with $n$ parallel copies of $(2,1)$ and $m-n$ parallel copies of $(0,1)$ is denoted $(2 n, m)$, demonstrating that $(0,1)$ and $(2,1)$ is a base.

We thus assume that $(0,1)$ and $(2,1)$ are 1-sided. Then any normalized surface can be denoted $(2 n, 2 m)$, where $n \leq 2 m$. For $m \geq n$, we have that $n$ parallel copies of $(2,2)$ union $m-n$ parallel copies of $(0,2)$ corresponds to $(2 n, 2 m)$. Hence assume that $m<n$. Then $2 m-n$ copies of $(2,2)$ union $n-m$ copies of $(4,2)$ corresponds to the surface $(2 n, 2 m)$. Thus $(0,2),(2,2)$, and $(4,2)$ constitute a base.

Case 1. [0,3] and [1,2].

Then any normalized surface can be denoted $(2 n, 2 m)$, where $n \leq 2 m$, so that $(0,2),(2,2)$, and $(4,2)$ constitute a base as per Case 0 .

Case 2. $\quad[0,3]$ and $[2,1]$.

The argument is identical to Case 0 .

Case 3. [1,2] and $[2,1]$.

Then both $\sharp\left(F \cap T_{1}^{0}\right)$ and $\sharp\left(F \cap T_{1}^{1}\right)$ must be even and non-zero, so that any normalized surface can be denoted $(2 n, 2 m), n, m>0$, with $n \leq 2 m$ and $m \leq 2 n$. For $m \geq n, 2 n-m$ copies of $(2,2)$ and $m-n$ copies of $(2,4)$ corresponds to $(2 n, 2 m)$. For $m<n, 2 m-n$ copies of $(2,2)$ and $n-m$ copies of $(4,2)$ corresponds to $(2 n, 2 m)$. Thus $(2,2),(2,4)$, and $(4,2)$ constitute a base. 
Case 4. [0,3], [1,2] and $[2,1]$.

The argument is identical to Case 3.

Case 5. [0,3], [1,2] and [3,0].

The argument is identical to Case 1.

Case 6. [0,3], [1,2], [2,1] and $[3,0]$.

The argument is identical to Case 3.

All other cases can be obtained from switching the roles of $T_{1}^{0}$ and $T_{1}^{1}$. This completes the proof.

Theorem 5.2. Let $M$ be a 3-manifold, and assume that $M$ has an IPD with $\sharp\left(T_{1}\right)=3$ such that any triangular face $T_{2}^{k}$ has at most two distinct edges. Then $(M, T)$ is good.

Proof. The proof, being analogous to 5.1 but having many more cases, is left to the reader. We note that two such manifolds are given in Examples 6.6 and 6.7.

Conjecture 5.3. Let $M$ be a 3-manifold, and assume that $M$ has an IPD such that any triangular face $T_{2}^{k}$ has at most two distinct edges. Then $(M, T)$ is good.

We note, for example, that Manifold $V_{3383}$, obtained from the cusped census of [11], and having a 7 tetrahedron decomposition, is an example of a manifold with a good IPD having $\sharp\left(T_{1}\right)=6$, see Figure 10. (It also has a good IPD with $\sharp\left(T_{1}\right)=7$.)

\section{Examples.}

We consider the cusped hyperbolic manifolds of small volume obtained from the cusped census of [11]. We give in Figure 11 a table of the triangulations and good IPD's we found for manifolds 000-006. Note that manifold 005 may have a good IPD. If so, it cannot be obtained from the canonical triangulation by gluing "bad" faces together.

If a Heegaard splitting of a manifold with a given IPD is just a regular neighborhood of (some boundary components union some components of $T_{1}$ ), then we say that the splitting is trivial (with respect to the IPD). In particular, when we examine some of the manifolds in the census of [11], we will often refer to the splittings as "trivial", the IPD being tacit. 
Note 6.0. In most cases, it is easy to check that Case 0 of 4.0 can be applied to any normalized surface with $\sharp(\bar{S})>2$. We shall tacitly eliminate all such cases. Further, we note that although we can color the pieces (as per 4.0) in two ways, we shall tacitly assume that we have chosen a coloring which eliminates application of Case 0 .

Example 6.1. Manifold 000, $M_{000}$, the Gieseking Manifold, has only one irreducible (non-orientable) Heegaard splitting.

This is just Example 6.0 of [6].

We note that the Gieseking Manifold is a nonorientable manifold which is double covered by the figure- 8 knot complement. The above result then implies that any Heegaard splitting surface for the figure- 8 knot complement which can be isotoped so as to be symmetric with respect to the double cover must be induced by the pull-back of the one splitting for $M_{000}$. It is elementary to check that this pulled-back surface is reducible.

Example 6.2. An irreducible Heegaard splitting $S$ of $M_{001}$ is trivial.

If $S$ is weakly rigid, then $S$ is trivial by 3.0. Otherwise, $S$ is Gabaic. The triangulation given in [11] has two edges, so it is good, having base $\{(2,0),(2,2)\}$, using the notation of 5.1 . Then to apply 4.0 we need only to check the normalized Gabai surfaces:

(a) $(4,0)$,

(b) $(4,2)$, and

(c) $(4,4)$.

For case (a), $\bar{H}_{0}$ is a non-trivial I-bundle over a surface of genus $\frac{3}{2}$ union a compression body, so Case 1 applies. Similarly for (b). For (c), $\bar{H}_{0}$ is again two pieces, one of which is homeomorphic to the original manifold. Thus Case 1 can again be applied.

Hence we must assume that $S$ is weakly reducible. All possible candidates for Haken surfaces are $(2,0)$ and $(2,2)$. It is easy to check that $(2,0)$ has an essential compressing disc. The surface $(2,2)$ is boundary parallel. Thus any non-trivial $S$ is almost trivial. But then it is easy to check (as per 6.1), that almost trivial $S$ are reducible, and hence stabilized.

Example 6.3. An irreducible Heegaard splitting $S$ of $M_{002}$ is trivial.

By 3.0 we assume that $S$ is not weakly rigid. Note that $M_{002}$ has two boundary components, $\partial_{0} M_{002}$, and $\partial_{1} M_{002}$. If $\left(\partial_{0} M_{002} \cup \partial_{1} M_{002}\right) \subset H_{1}$, then it is possible for $S$ to be non-Gabaic. But by reversing the flow of the Heegaard foliation (considering $1-h$ instead of $h$ ), we may assume that a non-weakly rigid Heegaard splitting of $M_{002}$ is Gabaic.

Again the triangulation is good, having base $\{(1,0),(1,2)\}$. We only need to check the Gabai surface $(2,4)$, since all other cases allow application of 
Case 0. But it is easy to check that Case 1 can be applied to this surface. Then, as per $M_{001}, S$ is trivial.

We note that $M_{002}$ is a nonorientable manifold which is double covered by the $6_{2}^{2}$-link complement. The above result implies that any Heegaard splitting surface for the link complement which can be isotoped so as to be symmetric with respect to the double cover must be induced by the pullback of one of the two splittings. It is elementary to check that both such splittings are reducible.

The $6_{2}^{2}$-link has 4 known Heegaard splittings of genus 2: Those given by the upper and lower tunnels, and those given by the two $\sigma$-tunnels (see [7]). It is interesting to note that the two (reducible) Heegaard splittings given by the above argument are: (1) that induced by the upper and lower tunnels (together), and (2) that induced by both $\sigma$-tunnels (together).

Example 6.4. An irreducible Heegaard splitting $S$ of either $M_{003}$ or $M_{004}$ is trivial.

We note that $M_{004}$ is the Figure- 8 Knot Complement, and $M_{003}$ is its so-called "Sister Manifold".

As per $M_{001}$, any such $S$ is either weakly rigid or Gabaic, the triangulations are good, and a base in either case is the set $\{(2,2),(2,4),(4,2)\}$. The surfaces we need to check are then:

(a) $(2,4)$ : Case 5 , weakly reducible,

(b) $(4,2)$ : Case 5, weakly reducible,

(c) $(4,4)$ : Case 1 ,

(d) $(4,6)$ : Case $4 ; \bar{H}_{1}$ compressible $\Rightarrow$ weakly reducible,

(e) $(4,8)$ : Case 4 ; construct $S$, easy to check weakly reducible,

(f) $(6,4)$ : as per $(d)$,

(g) $(8,4)$ : as per $(\mathrm{e})$.

Again as per the argument of $M_{001}$, weakly reducible implies reducible, so that $S$ must be trivial.

Conjecture 6.5. An irreducible Heegaard splitting $S$ of $M_{005}$ is trivial.

The author was unable to find a good IPD for this manifold, so this question remains open. However, even if a good IPD cannot be located, it may still be possible to prove that all Heegaard splittings of bounded genus are trivial. We note that in all cases we have checked, normalized Gabai surfaces have genus four or greater. If this can be proven to be always true, then since these surfaces have been obtained from compression of a Heegaard surface, any Heegaard splitting of genus 2 or 3 must be trivial or almost trivial. 
Example 6.6. An irreducible genus 2 Heegaard splitting $S$ of $M_{006}$ is trivial.

First note that $S$ must be weakly rigid or Gabaic. Also note that a good polyhedral decomposition for $M$ exists by identifying faces having 3 distinct edges, as per Figure 11.

We now use Lemma 3.4 on the good IPD, and apply the argument of 4.0 to the surfaces of genus less than 3 .

It is elementary to check that the set of normalized surfaces $\{(2,2,0)$, $(2,2,2),(2,2,4),(2,4,0),(2,4,2),(2,4,4),(4,2,0),(4,2,2),(4,2,4),(4,2,6),(4,2,8)$, $(8,4,2)\}$ forms a base for $T_{006} \cdot{ }^{1}$

We thus need to apply the argument of 1.0 to each of: $(2,2,4),(2,4,0)$, $(2,4,2),(2,4,4),(4,2,0),(4,2,2),(4,2,4)$, etc., that is, each of the Gabai surfaces not having three parallel copies of any base surface. Any of these of genus greater than 2 can be eliminated, since we are only interested in classifying the genus 2 case. This eliminates all of the cases except for $(2,4,2)$ and $(4,4,4)$.

$(\mathbf{2 , 4 , 2 )}$ : This is a surface of genus 2 , and divides $M$ into two pieces. One of the two pieces has first homology group $Z^{2} \oplus Z_{5}$, and thus cannot be a handlebody. Thus we can apply Case 3 of 4.0. It is a simple matter to continue the arguments to demonstrate that any such Heegaard splitting must in fact be induced by two edges of the 1-skeleton. At any rate, any such Heegaard surface is of genus greater than 2 .

$(4,4,4)$ : The center black piece is homeomorphic to $M_{006}$, so that we may apply Case 1.

Note then that a non-boundary parallel Haken surface $F$ must be of genus 2 or greater, and that a Heegaard splitting obtained from amalgamations of Haken submanifolds must thus be of genus at least 4 .

Thus any genus 2 Heegaard splitting is trivial or almost trivial. It is an easy matter to check that almost trivial Heegaard splittings are reducible (and of genus 3).

We also note that we restricted ourselves to genus 2 only for convenience. It seems that, given a little patience and a lot of time, we could check each of the exceptional surfaces and classify all Heegaard splittings.

Example 6.7. Any unknotting tunnel for the $5_{2}$ knot is isotopic to one of the edges in the canonical triangulation of its complement.

Proof. It is elementary to check that $M_{015}$ is the $5_{2}$ knot complement, and

\footnotetext{
${ }^{1}$ We also note that we can apply Lemma 3.4 to the triangulation and glue the "bad" faces together afterwards. Applying 4.0 to the good IPD at this point allows us to eliminate several surfaces.
} 
that it has two different good IPDs which are equivalent by a homeomorpism rotating the knot 180 degrees. Any unknotting tunnel for the knot is induced by a genus 2 Heegaard splitting. We thus classify all genus 2 Heegaard splittings, as per Example 6.6. There is only one Gabaic surface which is genus 2; it is shown after an isotopy in Figure 12. Although this is not induced by an edge in the IPD shown, it is easy to check that its spine is induced by an edge in the canonical triangulation. It in fact is a Heegaard surface for $M_{015}$. All other genus 2 Heegaard splittings must be rigid, hence induced by the 1-skeleton of the IPD, which coincides with the the other three edges of the canonical triangulation.

This is the desired result.

Example 6.8. The canonical triangulation of the Whitehead link complement, $W$.

The Whitehead link complement has canonical triangulation as shown in Figure 13. We call the boundary components $\partial_{0} W$ and $\partial_{1} W$. Let $S$ be a Heegaard splitting surface such that $\partial_{i} W=\partial_{-} H_{i}$. Then $T_{1}^{0}$ has an odd number of maxima, while $T_{1}^{1}$ has an odd number of minima. Assume that both have exactly one critical point. Then the Heegaard splitting is neither weakly rigid nor Gabaic. In addition, reversing the flow of the Heegaard foliation (switching $H_{0}$ and $H_{1}$ ) doesn't make the splitting weakly rigid or Gabaic, as per Example 6.3. Thus we cannot apply the results of this paper to this polyhedral decomposition of $W$. (We should also note that the canonical triangulation of $W$ is not "good".) 

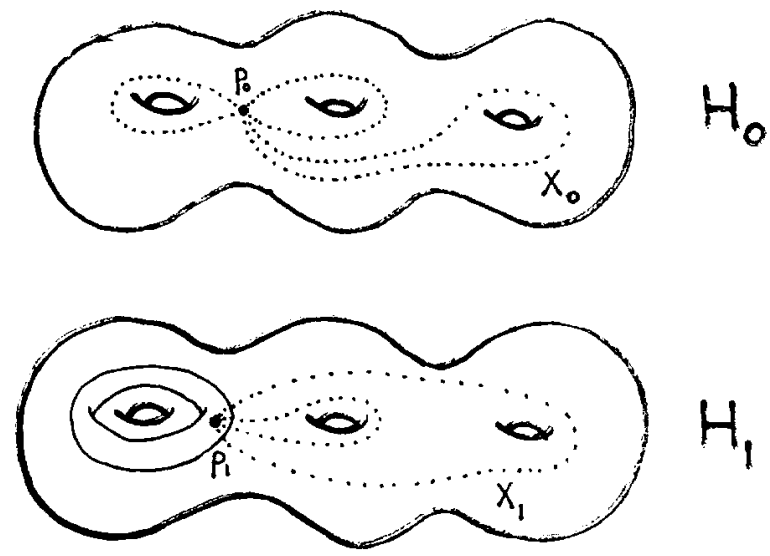

spinal complexity $\{2,3\}$
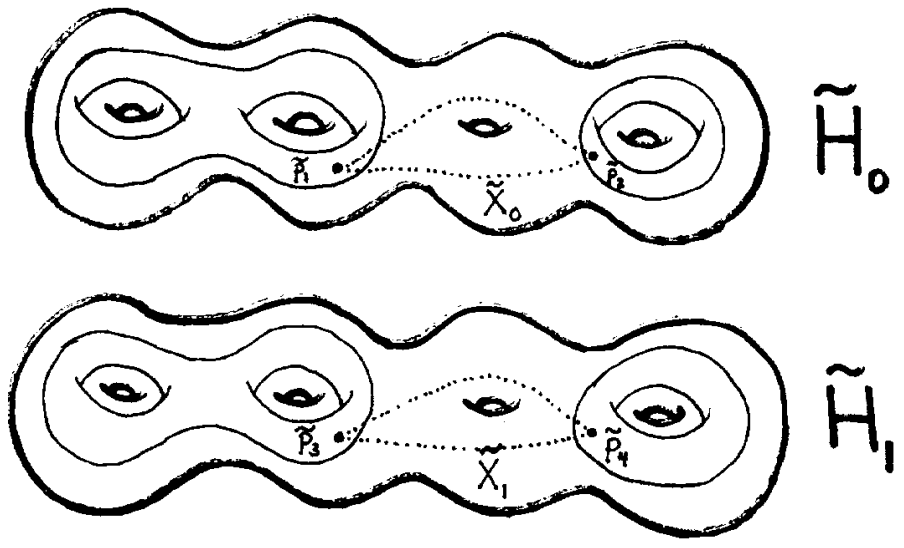

$$
\begin{gathered}
\text { spinal complexity }\{2,2\} \\
\{2,2\}<\{2,3\}
\end{gathered}
$$

Figure 0. 


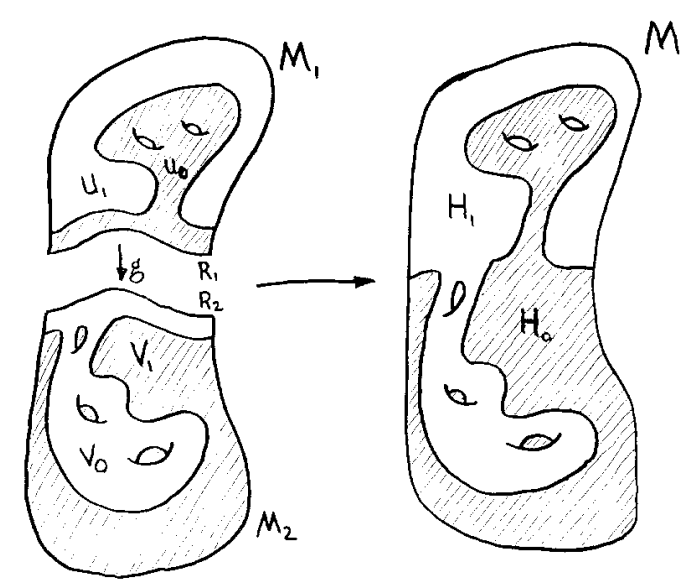

Figure 1.

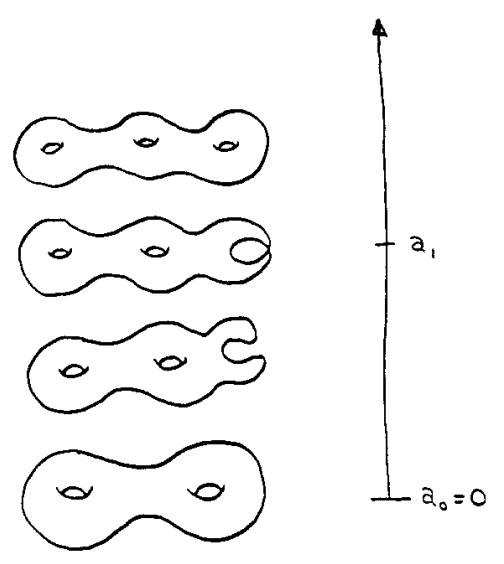

Figure 2.

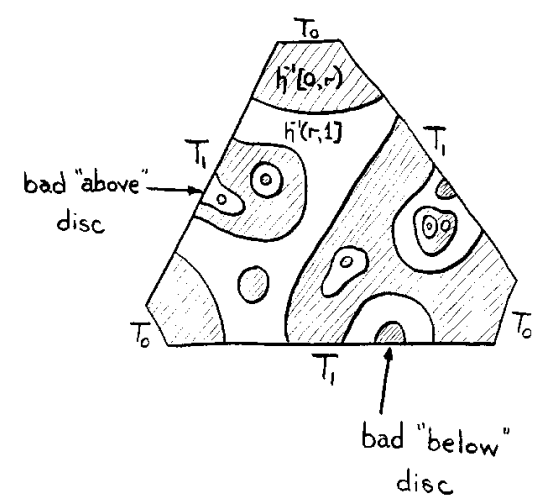

Figure 3. 


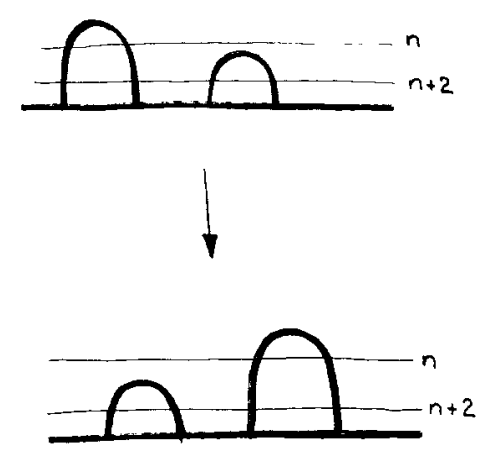

Figure 4.

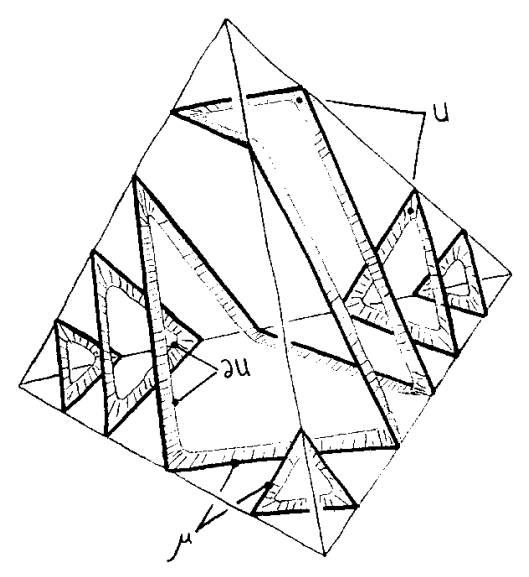

Figure 5.

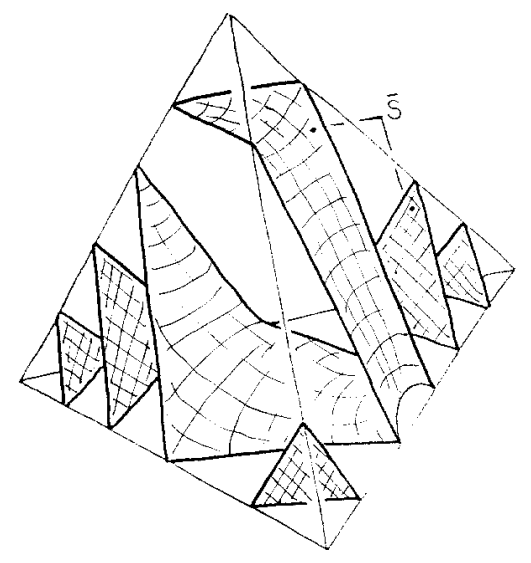

Figure 6. 


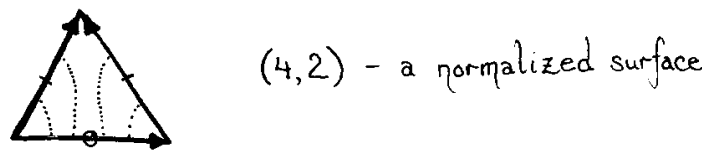

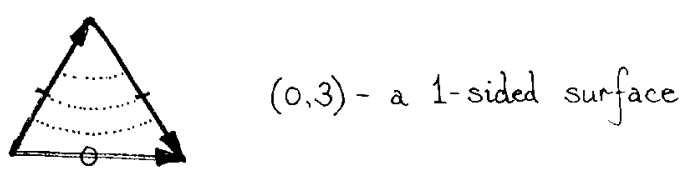

$(4,1)-$ no such normalized surface

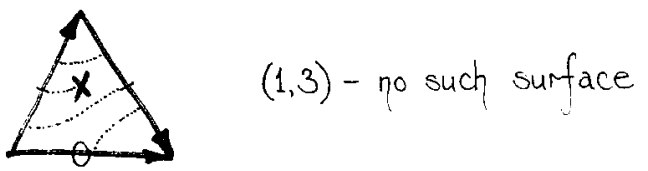

Figure 7 .
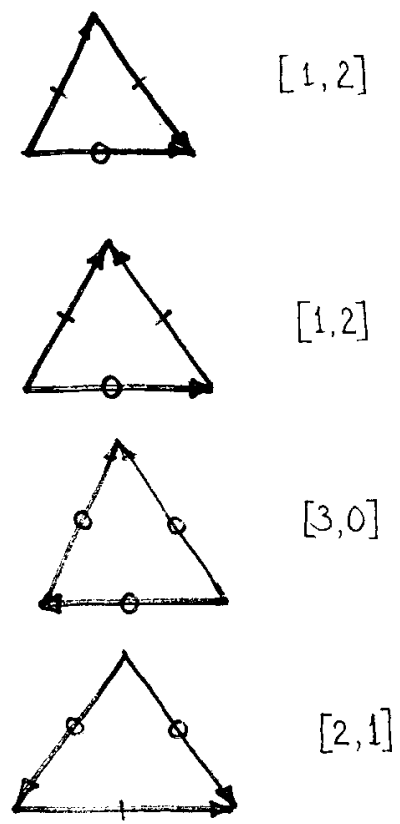

Figure 8. 


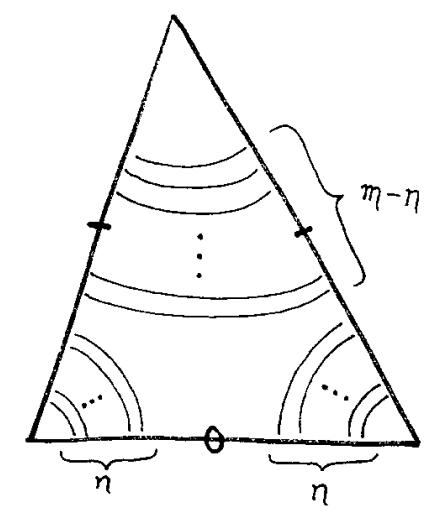

Figure 9.
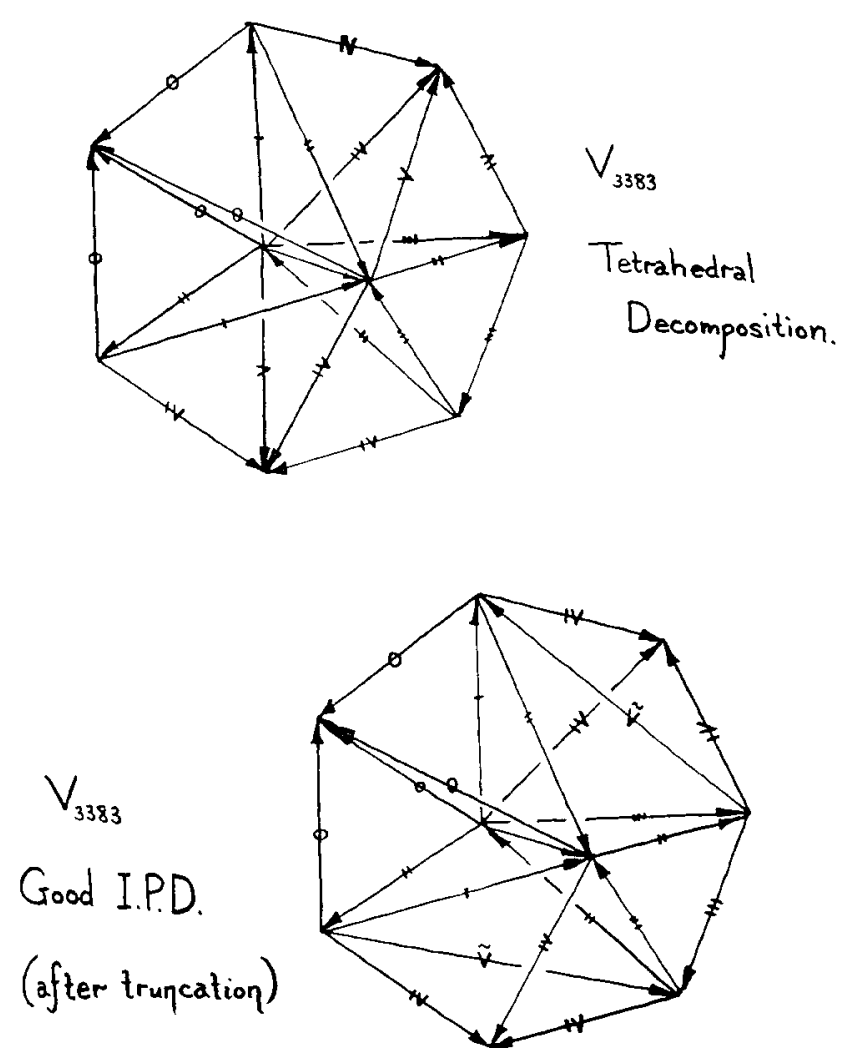

Figure 10. 


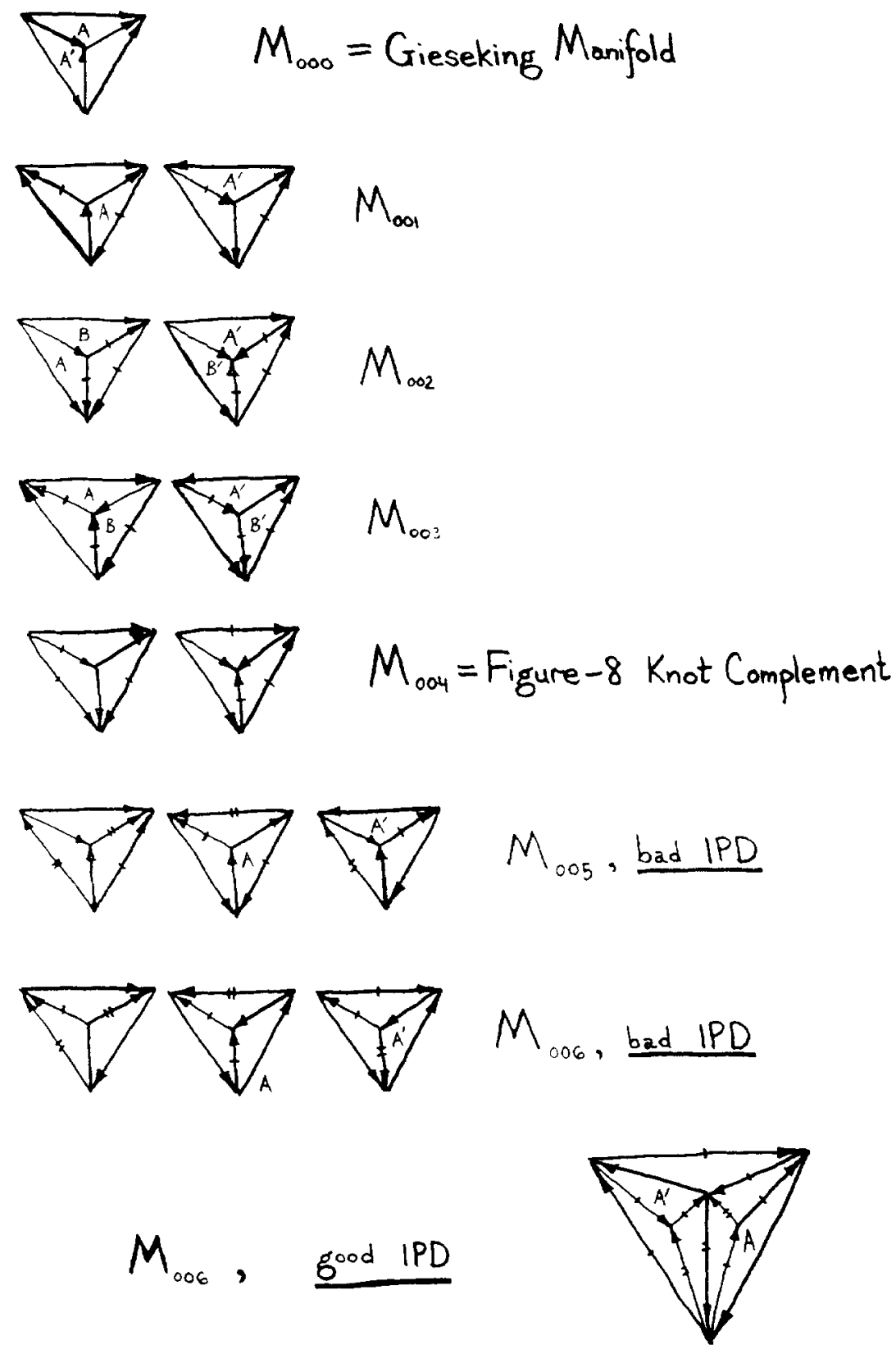

Figure 11. 


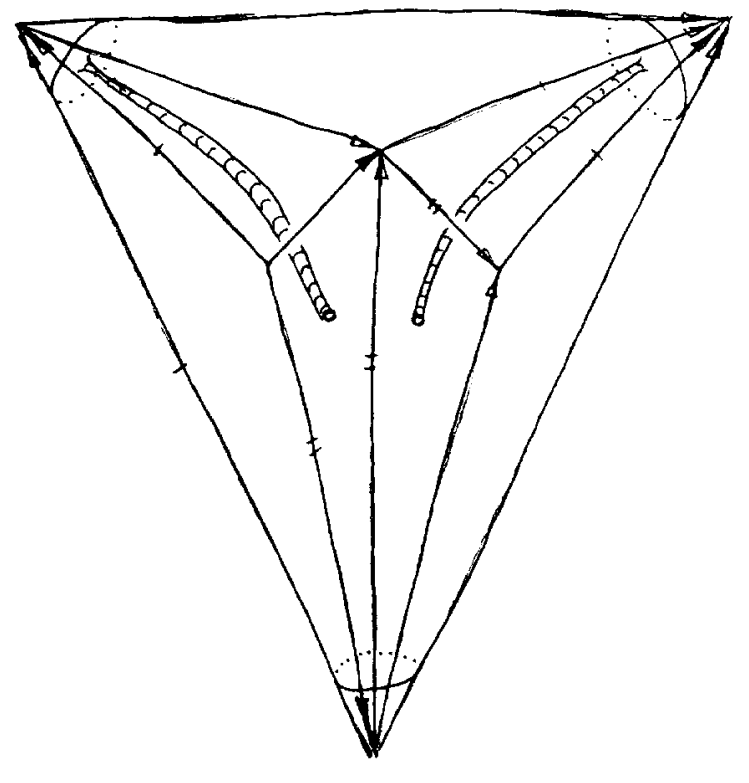

Figure 12.
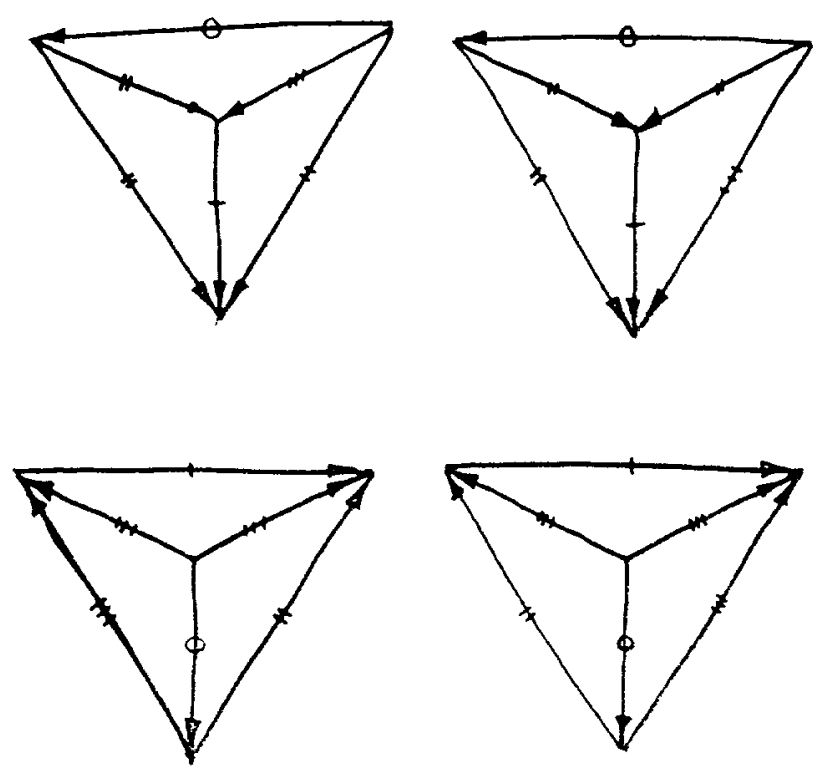

Figure 13. 


\section{References}

[1] F. Bonahon and J.P. Otal, Scindements de Heegaard des Espaces Lenticulaires, Ann. Sci. Ecole Norm. Sup., 16(4) (1983), no. 3, 451-466.

[2] A. Casson and C. Gordon, Reducing Heegaard Splittings, Topology and its applications, 27 (1987), 275-283.

[3] D. Gabai, Foliations and the Topology of 3-Manifolds, J. Diff. Geom., 18, 26 (1983, 1987) 445-503, 461-536.

[4] D. Heath, A Classification Theorem for Rigid Heegaard Splittings, preprint.

[5] - Heegaard Splittings of I-bundles and $\mathbf{R} P^{2} \times S^{1}$ are Standard, Dissertation.

[6] _ Heegaard Splittings of 1-edged Manifolds, preprint.

[7] T. Kobayashi, A criterion for detecting inequivalent tunnels for a knot, Math. Proc. Camb. Phil. Soc., 107 (1990) 483-491.

[8] K. Morimoto and M. Sakuma, On Unknotting Tunnels for Knots, Math. Ann., 289 (1991), 143-167.

[9] M. Scharlemann and A. Thompson, Heegaard Splittings of (orientable surface) $\times I$ are Standard, preprint.

[10] F. Waldhausen, Heegaardzerlegungen der 3-Sphaere, Topology, 7 (1968), 195-203.

[11] J. Weeks, Snappea, Computer program, available through the Geometry Center at the University of Minnesota.

Received July 18, 1995 and revised March 13, 1996.

TeXas A \& M University-Commerce

Commerce, TX 75429 